Ann. Génét. Sél. anim., 1981, 13 (2), 165-176

\title{
Variabilité génétique de la performance de nage chez l'alevin de Truite fario (Salmo trutta)
}

\author{
J.M. BLANC et J.F. TOULORGE \\ Laboratoire d'Ecologie des Poissons et d'Aménagement des Pêches \\ Centre de Recherches hydrobiologiques \\ Saint-Pée-sur-Nivelle, F 64310 Ascain
}

\begin{abstract}
Résumé
L'analyse de la variation de la performance de nage chez les alevins de 50 familles de Truite fario élevés en pisciculture met en évidence l'action simultanée de facteurs environnementaux collectifs (entre lots de même famille) et individuels (intra-lots), et de facteurs génétiques sélectionnables (entre familles) relativement peu importants (héritabilité estimée 0,3 ).

On observe toutefois que la performance de nage est liée à la taille des individus (corrélation $+0,5)$. L'ajustement des données à un modèle de régression améliore l'estimation relative des effets génétiques qui apparaissent assez spécifiques de l'aptitude à la nage, ce qui n'est pas le cas des effets environnementaux.

Ces résultats permettent d'envisager l'utilisation de la sélection familiale pour améliorer ce caractère dans les populations destinées aux repeuplements. Ils incitent par ailleurs à la prudence dans les tests de pollution basés sur la mesure de performances de nage.
\end{abstract}

\section{Introduction}

L'utilisation de la performance de nage des poissons pour apprécier leurs aptitudes constitutionnelles ou leur état physiologique a fait l'objet d'un nombre important de travaux (Thомas, 1970). Cette méthodologie a permis de mieux connaître les besoins physiologiques des espèces étudiées (BRETT \& GLASs, 1973 ; KuTTY \& SAUNDERS, 1973), et a donné lieu à deux applications principales : D'une part, la mise au point d'épreuves de nage pour apprécier la qualité des Salmonidés de repeuplement (Thomas et al., 1964 ; Burrows, 1969), et d'autre part le développement de bioessais destinés à la détection et à la mesure des polluants aux concentrations subléthales (Mc LEOD, 1967).

Toutefois, les facteurs génétiques susceptibles d'influencer les performances de nage n'ont été que rarement pris en considération, bien que des différences entre populations aient été mises en évidence chez l'Omble de fontaine (VINCENT, 1960) et chez la Truite arc-en-ciel (Thomas \& Donahoo, 1977 ; TsuYuKI \& Williscroft, 1977). 
Dans la présente étude, nous avons cherché à apprécier l'importance des effets génétiques sélectionnables sur la performance de nage, à l'intérieur d'une population d'alevins de Truite fario (Salmo trutta L.).

\section{Matériel et méthodes}

\section{Structure de la population expérimentale et conditions d'élevage}

La population expérimentale, destinée par ailleurs à une étude des paramètres génétiques de la croissance, était constituée de 50 familles de demi-frères-sœurs représentant un plan d'évaluation sur descendance de 50 mâles (croisés avec un ensemble de femelles), réalisé à la pisciculture de Saint-Pée-sur-Nivelle (PyrénéesAtlantiques) à l'aide de reproducteurs (mâles de 2 ans, femelles de 3 ans) choisis au hasard dans la souche de la pisciculture.

Après un mois d'incubation en milieu homogène (sur des clayettes compartimentées dans une eau filtrée à la température de $8^{\circ} \mathrm{C}$ ), les œufs prêts à éclore ont été transférés dans un ensemble d'auges aménagées en 150 compartiments (trois par famille) de même taille (fond : $80 \mathrm{~cm} \times 42 \mathrm{~cm}$; hauteur d'eau : $12 \mathrm{~cm}$ ) et alimentés avec la même eau (eau de la Nivelle filtrée). Ces installations étaient situées dans un bâtiment expérimental, donc à l'abri des perturbations atmosphériques.

Les épreuves de nage y ont été effectuées sur les alevins âgés de 4 mois et ayant atteint une longueur moyenne de $4,7 \mathrm{~cm}$.

\section{Conditions expérimentales}

La performance de nage a été mesurée par le temps de résistance des sujets dans un dispositif à courant d'eau rectiligne de vitesse uniformément croissante. L'appareil utilisé comportait (Fig. 1) :

- un tunnel de nage en verre de $100 \mathrm{~cm}$ de longueur et de $5 \mathrm{~cm}$ de diamètre intérieur, grillagé à l'extrémité amont, et se terminant à l'aval par un module destiné à l'introduction des alevins. A la jonction du tunnel et du module, une barrière électrique était alimentée en courant continu de 15 volts, lorsque le sujet avait pénétré dans le tunnel ;

- une pompe alimentant le tunnel de nage à partir d'un bac à niveau d'eau constant ; cette pompe était commandée par un variateur de vitesse permettant une augmentation linéaire de la vitesse du courant de 6,6 à $41 \mathrm{~cm} / \mathrm{s}$ sur une durée de 2'35" ; l'eau était renouvelée en permanence dans le système, à raison de $101 / \mathrm{mn}$ environ à partir des installations d'élevage, sans régulation thermique.

300 alevins, à raison de 2 choisis au hasard dans chaque compartiment d'élevage (soit 6 par famille) ont été examinés individuellement à l'aide de cet appareillage, dans un ordre établi par tirage au sort; le tunnel de nage étant recouvert d'un voile noir, l'individu introduit était d'abord laissé au repos pendant $2 \mathrm{mn}$ dans le courant faible $(6,6 \mathrm{~cm} / \mathrm{s})$ puis soumis à l'épreuve proprement dite en courant accéléré, le chronomètre étant déclenché en même temps que le variateur de vitesse. Du fait de 
son rhéotaxisme positif et de l'action de la barrière électrique, l'alevin tendait à se maintenir dans le tunnel jusqu'à épuisement. Le temps de nage était noté lorsque l'alevin se laissait enfin entraîner hors du tunnel.

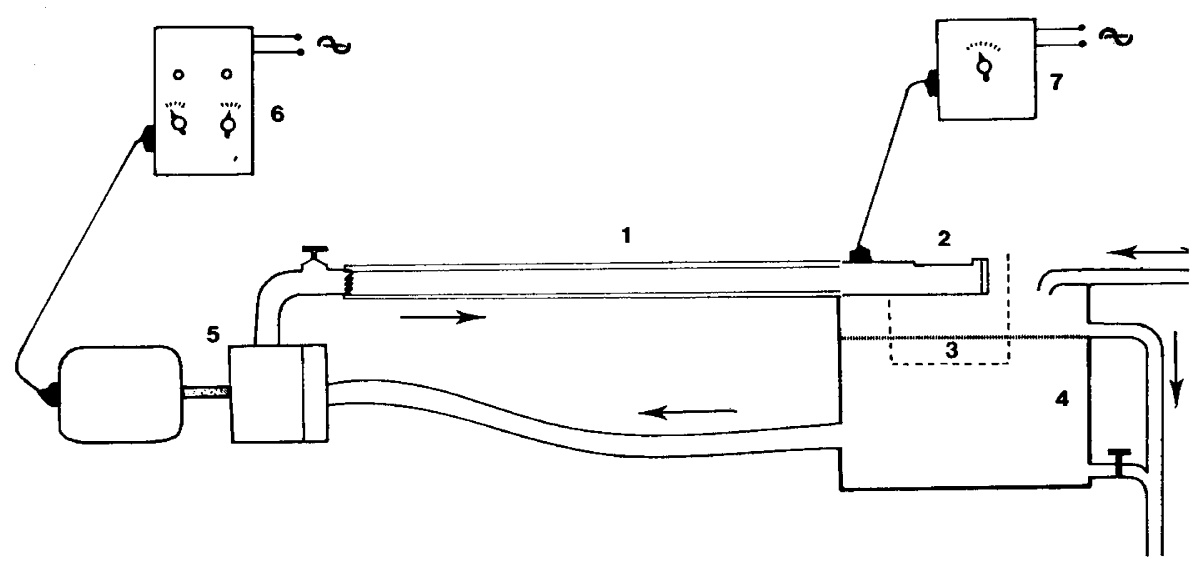

FIG. 1

Schéma de l'appareil de nage

1. Tunnel de nage

Diagram of the swimming apparatus

2. Module d'introduction des alevins

3. Panier de récupération

4. Bac réservoir avec surverse

5. Pompe

6. Variateur de vitesse

7. Commande de la barrière électrique

Immédiatement après l'épreuve, l'alevin était anesthésié et sa longueur corporelle mesurée. On notait en outre l'heure de la journée et la température de l'eau à la sortie du tunnel de nage.

Ces opérations ont duré 4 jours, de 8 heures à midi et de 14 heures à 18 heures. Pendant cette période, la température a varié entre 12,6 et $14,1^{\circ} \mathrm{C}$.

\section{Méthodes statistiques}

Les tests d'indépendance des performances de nage vis-à-vis de l'heure de la journée d'une part, et vis-à-vis de la température d'autre part, ont été effectués en classant les données sous forme de tableaux de contingence (4 classes de performances $\times 10$ classes horaires, et 8 classes de performances $\times 4$ classes de température) et en utilisant sur les fréquences obtenues le test G (SoKal \& Rohlf, 1969). 
L'analyse des performances de nage ( $P$, en secondes) en fonction de la longueur des individus ( $\mathbf{L}$, en $\mathrm{mm}$ ) a nécessité, pour un meilleur ajustement linéaire, la transformation logarithmique des variables : $\mathrm{X}=100 \log _{10} \mathrm{~L}$ et $\mathrm{Y}=100 \log _{10}$ P. Les modèles d'analyse de variance utilisés sont :

et

$$
X_{i j k}=\mu+A_{i}+C_{i j}+E_{i j k}
$$

$$
Y_{i j k}=\mu^{\prime}+A_{i}^{\prime}+C_{i j}^{\prime}+E_{i j k}^{\prime} \text { dans lesquels : }
$$

$\mathrm{X}_{\mathrm{ijk}}$ et $\mathrm{Y}_{\mathrm{ijk}}$ sont les valeurs transformées de la longueur et de la performance chez l'individu $\mathrm{k}$ du compartiment d'élevage $\mathrm{j}$ attribué à la famille $\mathrm{i}$.

$\mu$ et $\mu^{\prime}$ sont les espérances de ces caractères

$A_{i}$ et $A_{i}^{\prime}$ sont les effets paternels (famille)

$C_{i j}$ et $C_{i j}$ sont les effets d'environnement de groupe (compartiment)

$E_{i j k}$ et $E_{i j k}^{\prime}$ sont les écarts résiduels à la moyenne de compartiment d'élevage.

Les méthodes d'estimation des composantes de variance et de covariance (modèle hiérarchique à effets aléatoires) ainsi que l'interprétation génétique sont tirées de BECKER (1967), la variance de l'effet paternel représentant le quart de la variance génétique additive selon le modèle classique de FALCONER (1960).

Enfin un ajustement au modèle de régression suivant a été réalisé :

$Y_{i j k}=\mu^{\prime}+\beta\left(X_{i j k}-\mu\right)+A^{\prime \prime}{ }_{i}+C^{\prime \prime}{ }_{i j}+E^{\prime \prime}{ }_{i j k}$ dans lequel :

$\beta$ est le coefficient de régression de $Y$ en $X$.

$A^{\prime \prime}{ }_{i}, C^{\prime \prime}{ }_{i j}$ et $E^{\prime \prime}{ }_{i j k}$ sont les effets indépendants de $X$.

L'analyse de covariance aboutissant à cet ajustement a été effectuée d'après la méthode décrite par SNedecor \& Cochran (1967).

\section{Résultats}

Les temps de nage des 300 individus étudiés se répartissent entre 30 et 144 secondes, valeurs extrêmes correspondant à des vitesses de courant limites de 13,3 et $38,6 \mathrm{~cm} / \mathrm{s}$. La performance moyenne est 68,7 secondes, soit une vitesse limite moyenne de $21,8 \mathrm{~cm} / \mathrm{s}$.

\section{Comparaison selon l'heure et la température}

La comparaison des performances enregistrées aux différentes heures de la journée ne met en évidence aucune évolution nyctémérale et le test d'indépendance $\mathrm{G}$ appliqué aux fréquences de classes aboutit au résultat : $\chi^{2}=27,6$ à 27 degrés de liberté, non significatif. 
De même, les performances ne semblent pas affectées par la température ce qui est confirmé par le résultat du test $\mathbf{G}$ :

$\chi^{2}=13,4$ à 21 degrés de liberté, non significatif.

Analyse conjointe de la performance de nage et de la longueur corporelle

Le nuage de points représentant graphiquement la correspondance entre la performance de nage et la longueur corporelle (en échelles logarithmiques, Fig. 2) fait apparaître une nette corrélation. On observe que la relation moyenne est approximativement linéaire, mais que les écarts de $\mathrm{Y}$ à la régression de $\mathrm{Y}$ en $\mathrm{X}$ ne sont pas normalement distribués (coefficient d'asymétrie : - 0,851 significatif à $\mathrm{p}=0,001$ ).

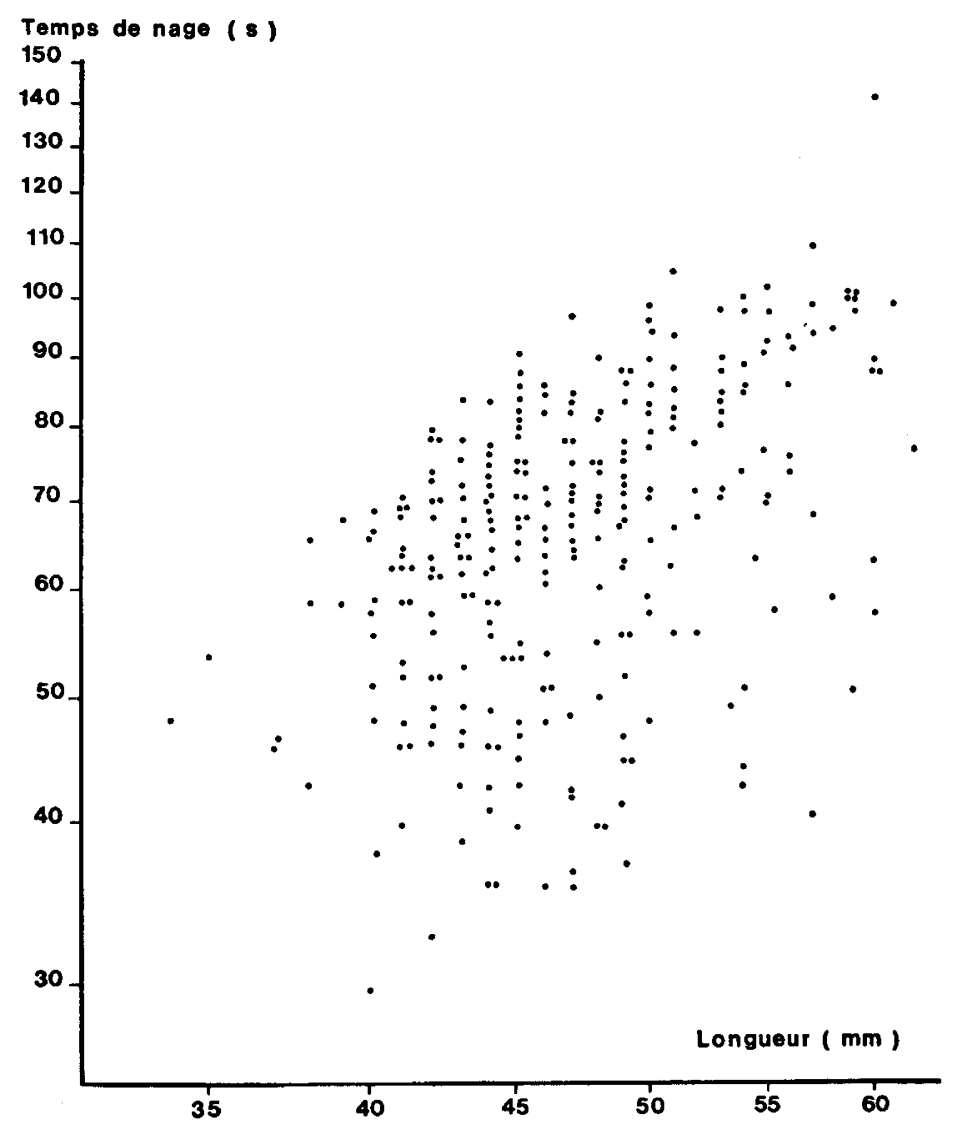

FIg. 2

Relation entre la performance de nage et la longueur corporelle (échelles logarithmiques) Relationship between swimming performance and body length (log scales) 
Les résultats de la décomposition des variances et de la covariance de $\mathrm{X}$ et $\mathrm{Y}$ sont présentés dans le tableau 1. Pour chacune des deux variables, les écarts entre familles ne sont pas significatifs au seuil $\mathrm{p}=0,05$, de même que les écarts entre compartiments intra-familles. Les estimations de la variance génétique additive $\left(\mathrm{V}_{\mathrm{G}}\right)$ et de l'héritabilité $\left(\mathrm{h}^{2}\right.$ : rapport de $\mathrm{V}_{\mathrm{G}}$ à la variance totale) n'ont donc qu'une valeur indicative :

$$
\begin{aligned}
& \text { pour } X: V_{G}=5,8 \text { d'où } h^{2}=0,24 \text { (écart-type d'erreur } 0,21 \text { ) } \\
& \text { pour } Y: V_{G}=33,4 \text { d'où } h^{2}=0,26 \text { (écart-type d'erreur } 0,21 \text { ) }
\end{aligned}
$$

Il apparaît d'autre part que la covariance est issue en presque totalité des effets de compartiments et résiduels. Par suite l'estimation de la corrélation génétique additive est faible $(0,22$, ne différant pas significativement de zéro) par rapport à la corrélation relative aux autres sources de variation (0,56 en moyenne, valeur hautement significative).

\section{TABLeaU 1}

Composantes de variances et de covariance relatives à la performance de nage et à la longueur corporelle

(les écarts-types d'erreur approximatifs sont indiqués entre parenthèses)

Variance and covariance components of swimming performance and body length

\begin{tabular}{|c|c|c|c|c|c|}
\hline \multirow{2}{*}{$\begin{array}{c}\text { Source } \\
\text { de variation }\end{array}$} & \multirow{2}{*}{$\begin{array}{l}\text { Degrés } \\
\text { de liberté }\end{array}$} & \multicolumn{2}{|c|}{ Composantes de variance } & \multirow{2}{*}{$\begin{array}{c}\text { Composante } \\
\text { de } \\
\text { covariance }\end{array}$} & \multirow[b]{2}{*}{ Corrélation } \\
\hline & & $\mathrm{X}$ (longueur) & $\begin{array}{l}\mathrm{Y} \text { (perfor- } \\
\text { mance) }\end{array}$ & & \\
\hline Familles . & 49 & $\begin{array}{c}1,45 \\
(1,23)\end{array}$ & $\begin{array}{c}8,36 \\
(6,91)\end{array}$ & $\begin{array}{c}0,75 \\
(2,27)\end{array}$ & $\begin{array}{c}0,22 \\
(0,56)\end{array}$ \\
\hline Compartiments & 100 & $\begin{array}{c}2,11 \\
(2,07)\end{array}$ & $\begin{array}{c}15,48 \\
(11,27)\end{array}$ & $\begin{array}{c}4,69 \\
(3,82)\end{array}$ & $\begin{array}{c}0,82 \\
(0,41)\end{array}$ \\
\hline Résidus & 150 & $\begin{array}{c}20,28 \\
(2,33)\end{array}$ & $\begin{array}{c}105,04 \\
(12,05)\end{array}$ & $\begin{array}{c}20,81 \\
(4,11)\end{array}$ & $\begin{array}{c}0,45 \\
(0,06)\end{array}$ \\
\hline Total . & & 23,84 & 128,88 & 26,25 & 0,47 \\
\hline
\end{tabular}
(approximate standard-error included in parentheses)

L'hypothèse que la longueur corporelle d'un individu est en rapport direct avec sa force physique dont dépend sa performance de nage conduit à rechercher un modèle de régression de $\mathrm{Y}$ en $\mathrm{X}$. Les calculs et résultats correspondants sont présentés, dans le tableau 2, en deux étapes :

- dans l'analyse intra-familles, les moyennes de compartiments ajustées sur la régression résiduelle ne diffèrent pas significativement; 


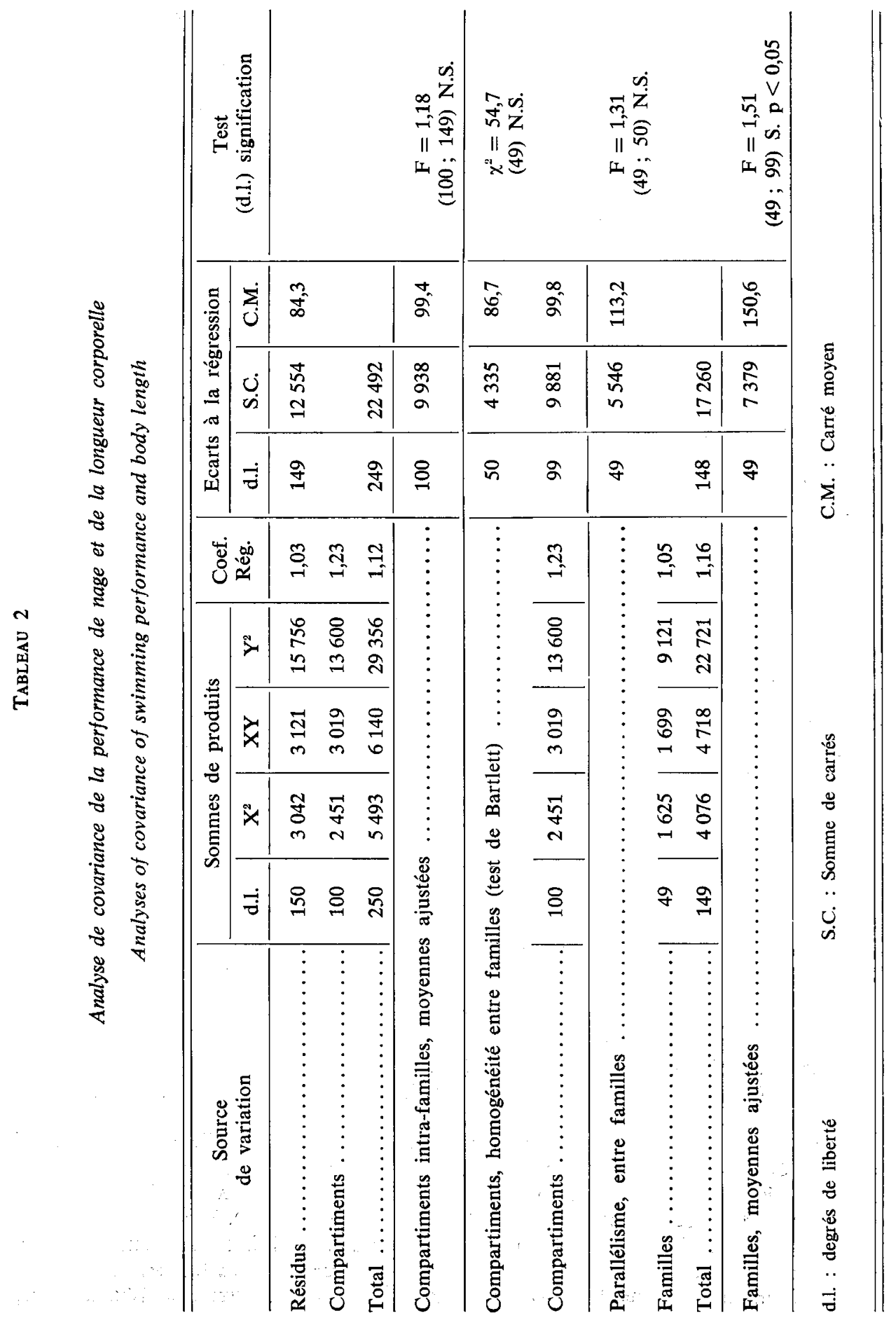


- dans l'analyse entre familles, les régressions calculées d'après les moyennes de compartiments montrent des variances liées semblables et des pentes semblables (tests non significatifs). Par contre les moyennes de familles, ajustées sur la régression moyenne des compartiments, montrent des écarts significatifs.

On constate enfin que les trois estimations indépendantes obtenues pour la pente de régression :

intra-compartiments, $\beta=1,03$, d'écart-type 0,17 ,

entre compartiments intra-familles, $\beta=1,23$, d'écart-type 0,20 ,

entre familles, $\beta=1,05$, d'écart-type 0,31 , ne diffèrent pas significativement entre elles.

Par suite il est possible d'estimer les paramètres du modèle :

$$
\mathbf{Y}_{\mathrm{ijk}}=\mu^{\prime}+\beta\left(\mathbf{X}_{\mathrm{ijk}}-\mu\right)+{A^{\prime \prime}}_{i}+C^{\prime \prime}{ }_{i j}+E^{\prime \prime}{ }_{i j k}
$$
de $X$ :

A savoir la régression pondérée $\beta=1,10$ et les variances des écarts indépendants

- écarts résiduels : $\mathrm{V}_{\mathrm{E}^{\prime \prime}}=84,4$

- effets de compartiments : $\mathrm{V}_{\mathrm{C}^{\prime \prime}}=7,4$ (non significative)

-- effets paternels: $\mathrm{V}_{\mathrm{A}^{\prime \prime}}=8,5$ (significative au seuil $\mathrm{p}=0,05$ ).

La comparaison de ces résultats avec ceux présentés dans le tableau 1 montre que la prise en compte de la régression a pour effet de réduire les variations non génétiques, mais laisse la variance entre familles sensiblement inchangée. L'héritabilité du caractère «performance de nage indépendante de la longueur » atteint de ce fait la valeur $h^{2}=0,34$ (estimation significativement différente de zéro, et dont l'écart-type d'erreur est environ 0,22).

\section{Discussion et conclusion}

Les nombreuses différences techniques existant entre les diverses méthodes de mesure de la performance de nage rendent difficiles les comparaisons entre les résultats, du moins en valeur absolue. Un certain nombre de recoupements peuvent néanmoins être effectués entre nos observations et celles publiées antérieurement.

La relation existant entre la performance de nage et la taille corporelle a été mise en évidence par de nombreux auteurs, la transformation logarithmique étant parfois utilisée pour le poids ou la longueur des individus (ThомAs et al., 1964), ainsi que pour leur vitesse maximum de nage (BRETT \& GLASS, 1973). Les vitesses atteintes par nos sujets expérimentaux (20 à 30 centimètres, soit 4 à 5 fois leur longueur, par seconde) sont du même ordre de grandeur, quoiqu'un peu plus faibles, que les performances mesurées au même âge chez la Truite arc-en-ciel (TsUYuKı \& WILLISCROFT, 
1977), le Saumon coho (Glova \& Mc InERney, 1977) et le Saumon sockeye (BretT \& Glass, 1973).

L'absence dans nos résultats de variation nyctémérale ou liée à la température de l'eau est à mettre en relation avec la stabilité assez bonne des conditions d'expérience : protection contre les rayons solaires directs, faible amplitude des variations thermiques et oxygénation constante assurée par le renouvellement de l'eau.

La mise en évidence d'une variation d'origine génétique entre les progénitures des 50 reproducteurs mâles utilisés constitue le principal résultat de cette étude. Cette variation apparaît pratiquement indépendante des facteurs génétiques influençant la croissance, constatation également faite par THOMAS \& DonaHOo (1977). L'estimation de la composante de variance correspondante et de son importance relative (héritabilité : environ 0,3 ) est néanmoins entachée d'une certaine imprécision due au faible effectif d'échantillonnage des alevins testés, compte tenu de l'importance de la variation résiduelle. A cela s'ajoutent des problèmes relatifs à la distribution de la variable étudiée, auxquels il faudrait porter attention dans le cadre d'une étude plus précise.

Il est d'autre part prudent de ne pas donner trop hâtivement à nos résultats une portée générale, étant donné que :

- les alevins utilisés n'avaient subi aucun entraînement physique au cours de leur élevage et étaient issus d'une souche de pisciculture susceptible d'avoir subi des modifications génétiques inhérentes à la domestication (VINCENT, 1960) ;

- la méthode employée mesurait la vitesse maximum de nage des sujets, plutôt que leur endurance, alors que ces deux caractères ne mettent pas en jeu les mêmes facteurs anatomiques et physiologiques (GATZ, 1973).

Ces réserves étant faites, l'existence d'une variabilité génétique de la performance de nage chez les Salmonidés aurait deux conséquences :

a) Dans la mesure où ce caractère serait en relation avec la qualité des sujets de repeuplement (BuRrows, 1969), il pourrait faire l'objet d'améliorations génétiques en pisciculture. Compte tenu de l'estimation relativement faible de l'héritabilité obtenue dans cette étude, les méthodes de sélection familiale seraient sans doute à préconiser, de préférence à la sélection individuelle. Un doute sérieux existe toutefois quant à la corrélation entre la performance de nage et la survie dans la nature (HoRAK, 1972) et des études supplémentaires apparaissent nécessaires avant d'engager des opérations importantes d'amélioration génétique.

b) Si l'utilisation de l'abaissement relatif de la performance de nage pour la mesure du degré de nocivité des pollutions à des doses subléthales devait se développer à l'avenir (LESEL, 1976), il serait souhaitable d'éviter que les variations génétiques ne constituent une source de variabilité incontrôlée. La création de souches de référence stables et aussi homogènes que possible destinées à ces tests d'une part, et l'élaboration de plans d'expérimentation génétique sur ce sujet d'autre part, pourraient apporter des solutions à ce problème. 


\section{Remerciements}

Nous remercions R. Lesel qui nous a prêté l'appareil de testage utilisé dans cette étude, ainsi que $\mathbf{J}$. Y YNCI pour son assistance technique. L'analyse des données a été réalisée avec le matériel de calcul du Centre de Recherches de Bordeaux, I.N.R.A., et l'assistance technique de C. Boussion. Nous remercions enfin B. Chevassus et P. Decombe qui nous ont conseillés pour la présentation des résultats.

\section{Summary}

Genetic variability of the swimming performance of Brown Trout alevin (Salmo trutta)

An analysis of the variaticn of swimming performance among hatchery-raised alevins from 50 families of Brown Trout shows evidence of the simultaneous action of common (between lots of same family) and individual (within-lots) environmental factors, and of additive genetic factors (beiwen families) which are of little relative importance (estimated heritability 0.3 ).

It is however observed that swimming performance is related to the size of individuals (correlation coefficient - -0.5 ). An adjustment of data to a regression model improves the relative estimation of genelic effects, which appear to be rather specific of swimming ability, while it is not the case for environmental effects.

These results allow considering the use of family selection for the improvement of this character in populations raised for stocking. They also suggest some caution in the design of pollution tests based on the measurement of swimming performances.

\section{Références bibliographiques}

BeCKer W.A., 1967. Manual of procedures in quantitative genetics. Washington State Univ. Press (2nd ed.), Pullman, Was., $130 \mathrm{p}$.

BretT J.R., Glass N.R, 1973. Metabolic rates and critical swimming speeds of sockeye salmon (Oncorhynchus nerka) in relation to size and temperature. J. Fish. Res. Bd Can., 30, 379-387.

Burrows R.E., 1969. The influence of fingerling quality on adult Salmon survivals. Trans. Amer. Fish. Soc., 99, 777-784.

FAICONER D.S., 1960. Introduction to quantitative genetics. Oliver and Boyd Ed., Edinburgh, $365 \mathrm{p}$.

Gatz A.J. Jr., 1973. Speed, stamina and muscles in fishes. J. Fish. Res. Bd Can., 30, 325-328.

Glova G.J., MC InERNEy J.E., 1977. Critical swimming speeds of coho Salmon (Oncorhynchus kisutch) fry to smolt stages in relation to salinity and temperature. J. Fish. Res. Bd Can., 34, 151-154.

HoRak D.L., 1972. Survival of hatchery-reared rainbow trout (Salmo gairdneri) in relation to stamina tunnel ratings. J. Fish. Res. Bd Can., 29, 1005-1009.

KuTTY M.N., SAunderS R.L., 1973. Swimming performance of young atlantic Salmon (Salmo salar) as affected by reduced ambient oxygen concentration. J. Fish. Res. Bd Can., 30, 223-227.

LESEr. R., 1976. Relationship between laboratory bioassays and in situ experiments p. 301-308. In Principles and methods for determining ecological criteria on hydrobiocenoses (Commission of the European Communities). Pergamon Press, Oxford. 
MC LEOD J.C., 1967. A new apparatus for measuring maximum swimming speeds of small fish. J. Fish. Res. Bd Can., 24, 1241-1252.

Snedecor G.W., Cochran W.G., 1967. Statistical methods. Iowa State Univ. Press, Ames, Iow., $593 \mathrm{p}$.

Sokal R.R., Rohlf F.J., 1969. Biometry, Freeman, San Francisco, Cal., 776 p.

Thomas A.E., Burrows R.E., Chenoweth H.H., 1964. A device for stamina measurement of fingerling salmonids. U.S. Fish Wildl. Serv. Res. Rep., 67, 1-15.

Thомаs A.E., 1970. An annotated bibliography on exercise, stamina, and swimming ability in fishes. A working paper of the workshop on salmon and trout stamina, December 2, 1970, at Portland, Oregon, U.S.A., 373 p.

Thomas A.E., Donahoo M.J., 1977. Differences in swimming performance among strains of rainbow trout (Salmo gairdneri). J. Fish. Res. Bd Can., 34, 304-306.

Tsuyuki H., Williscroft S.N., 1977. Swimming stamina differences between genotypically distinct forms of rainbow (Salmo gairdneri) and steelhead trout. J. Fish. Res. Bd Can., 34, 996-1003.

VINCENT R.E., 1960. Some influences of domestication upon three stocks of brook trout (Salvelinus fontinalis Mitchill) Trans. Amcr. Fish. Soc., 89, 35-52. 